\title{
The Existence of a Second Vesicular Glutamate Transporter Specifies Subpopulations of Glutamatergic Neurons
}

\author{
Etienne Herzog, ${ }^{1}$ Gian Carlo Bellenchi, ${ }^{2}$ Christelle Gras, ${ }^{1}$ Véronique Bernard, ${ }^{3}$ Philippe Ravassard, ${ }^{4}$ \\ Cécile Bedet, ${ }^{2}$ Bruno Gasnier, ${ }^{2}$ Bruno Giros, ${ }^{1}$ and Salah EI Mestikawy ${ }^{1}$ \\ 1/nstitut National de la Santé et de la Recherche Médicale U513, Faculté de Médecine, 94010 Créteil Cedex, France, \\ ${ }^{2}$ Centre National de la Recherche Scientifique (CNRS) Unité Propre de Recherche 1929, Institut de Biologie Physico- \\ Chimique, 75005 Paris, France, ${ }^{3}$ CNRS Unité Mixte de Recherche 5541, Université Bordeaux 2, 33076 Bordeaux Cedex, \\ France, and ${ }^{4}$ CNRS Formation de Recherche en Evolution 2360, Hôpital Pitié-Salpétrière, 75013 Paris, France
}

\begin{abstract}
Before their exocytotic release during stimulation of nerve terminals, nonpeptide neurotransmitters are loaded into synaptic vesicles by specific transporters. Recently, a protein initially identified as brain-specific $\mathrm{Na}^{+}$-dependent inorganic phosphate transporter I (BNPI) has been shown to represent a vesicular glutamate transporter (VGLUT1). In this study, we investigated whether a highly homologous "differentiationassociated $\mathrm{Na}^{+}$-dependent inorganic phosphate transporter" (DNPI) is involved in glutamatergic transmission. Vesicles isolated from BON cells expressing recombinant DNPI accumulated L-glutamate with bioenergetical and pharmacological characteristics identical to those displayed by VGLUT1 and by
\end{abstract}

brain synaptic vesicles. Moreover, DNPI localized to synaptic vesicles, at synapses exhibiting classical excitatory features. DNPI thus represents a novel vesicular glutamate transporter (VGLUT2). The distributions of each VGLUT transcript in brain were highly complementary, with only a partial regional and cellular overlap. At the protein level, we could only detect either VGLUT1- or VGLUT2-expressing presynaptic boutons. The existence of two VGLUTs thus defines distinct subsets of glutamatergic neurons.

Key words: glutamate; neurotransmitter transporter; synaptic vesicle; excitatory neurotransmission; DNPI; BNPI
Nonpeptide neurotransmitters are stored into synaptic vesicles by specific transporters driven by a V-type $\mathrm{H}^{+}$-ATPase (Gasnier, 2000). Two recent studies have identified a transporter responsible for the vesicular storage of glutamate (Bellocchio et al., 2000; Takamori et al., 2000), the major excitatory transmitter of mammalian CNS. Surprisingly, this protein was initially described as a brain-specific $\mathrm{Na}^{+}$-dependent inorganic phosphate (Pi) transporter I (BNPI) based on its induction of an $\mathrm{Na}^{+} / \mathrm{Pi}$ cotransport when expressed in Xenopus oocytes (Ni et al., 1994). However, an impressive set of data now advocates a role in the filling of glutamatergic vesicles. First, EAT-4, a Caenorhabditis elegans homolog of BNPI, is specifically involved in glutamatergic neurotransmission at the presynaptic level (Dent et al., 1997; Lee et al., 1999). Second, BNPI localizes to a subset of glutamatergic synaptic vesicles (Bellocchio et al., 1998). Third, the expression of BNPI in neuroendocrine cells induces a glutamate uptake activity that shares all of the characteristics observed on synaptic vesicles (Bellocchio et al., 2000; Takamori et al., 2000). Fourth, $\mathrm{BNPI}$ induces a quantal release of glutamate in a serotoninergic

Received July 6, 2001; revised Aug. 17, 2001; accepted Aug. 29, 2001.

This research was supported by grants from Institut National de la Santé et de la Recherche Médicale (B. Giros) and Centre National de la Recherche Scientifique. E.H. received a grant from the French Ministry of Research. G.C.B. was supported by European Community Training and Mobility for Researchers Program Contract FMRX-CT98-0228. We are indebted to B. Wiedenmann for giving the BON cell line, M. F. Isambert for designing the cell nebulization procedure, E. Doudnikoff and L. Grattier for technical support on electronic microscopy, P. Dreyfus for his patient and efficient help with the confocal microscope, and J. P. Henry for fruitful discussions.

E.H. and G.C.B. contributed equally to this work.

Correspondence should be addressed to Dr. Salah El Mestikawy, Institut National de la Santé et de la Recherche Médicale U513, Faculté de Médecine, 8 rue du Général Sarrail, 94010 Créteil Cedex, France. E-mail: salah.elmestikawy@im3.inserm.fr.

Copyright () 2001 Society for Neuroscience $\quad 0270-6474 / 01 / 210001-06 \$ 15.00 / 0$ cell line (Takamori et al., 2000). Last, autaptic GABAergic neurons expressing recombinant BNPI display an additional glutamatergic response (Takamori et al., 2000). BNPI is thus not only necessary but is sufficient for the exocytotic release of glutamate. Accordingly, the protein was renamed VGLUT1 (vesicular glutamate transporter 1).

VGLUT1 belongs to the type 1 family of $\mathrm{Na}^{+} / \mathrm{Pi}$ cotransporters (Werner et al., 1998). A "differentiation-associated $\mathrm{Na}^{+}$dependent inorganic phosphate transporter" (DNPI) that is upregulated during the differentiation of rat pancreatic AR42J cells into neuron-like cells was described recently in this family (Aihara et al., 2000). Interestingly, DNPI is highly homologous to VGLUT1 (82\% amino acid identity), and its transcript is abundant in brain regions that apparently lack VGLUT1 mRNA. Moreover, DNPI mRNA localizes to neurons (Hisano et al., 2000). Recent studies have established a vesicular localization of DNPI in excitatory neurons (Fujiyama et al., 2001; Sakata-Haga et al., 2001). We thus decided to examine the hypothesis that DNPI might represent a novel vesicular glutamate transporter.

This article is published in The Journal of Neuroscience, Rapid Communications Section, which publishes brief, peerreviewed papers online, not in print. Rapid Communications are posted online approximately one month earlier than they would appear if printed. They are listed in the Table of Contents of the next open issue of JNeurosci. Cite this article as: JNeurosci, 2001, 21:RC181 (1-6). The publication date is the date of posting online at www.jneurosci.org.

http://www.jneurosci.org/cgi/content/full/5807 


\section{MATERIALS AND METHODS}

$c D N A$ cloning and expression. The DNPI full-length clone was obtained by screening a $\lambda$ Z ap II hippocampus cDNA library (Stratagene, La Jolla, CA) with a probe derived from the published sequence (nucleotides 356-924) (Aihara et al., 2000). This resulted in the isolation of a $3000 \mathrm{bp}$ cDNA insert in pBluescript. VGLUT1 was isolated by reverse transcription (RT)-PCR amplification of total rat brain RNA using the access system (Promega, Madison, WI) with primers 5'-GGCACAGCCCACCATGGAGTTC-3' (forward) and 5'-TGAGGCAGGAGAGGAGTGGG-3' (reverse) and total rat brain RNA. The PCR product was cloned into pCRII-Topo (Invitrogen, San Diego, CA). Both inserts were sequenced and subcloned into the expression vector pcDNA3 (Invitrogen).

The BON cell line (a gift from B. Wiedenmann, Berlin, Germany) was cultured at $37^{\circ} \mathrm{C}$ under $5 \% \mathrm{CO}_{2}$ in DMEM-nutrient mix F-12 (1:1) (Life Technologies, Gaithersburg, MD), supplemented with $7.5 \%$ fetal bovine serum, $100 \mathrm{U} / \mathrm{ml}$ penicillin, and $100 \mathrm{mg} / \mathrm{ml}$ streptomycin. Cells were transfected by electroporation with pcDNA3 vectors (Invitrogen) bearing the rat DNPI, VGLUT1, or VIAAT (vesicular inhibitory amino acid transporter, also termed VGAT) (E. Herzog, unpublished observation) cDNAs or no insert. Stable transfectants were selected with $800 \mu \mathrm{g} / \mathrm{ml}$ G418 (Life Technologies) and screened by immunofluorescence and immunoblotting.

Subcellular fractionation and amino acid uptake assay. Synaptosomes and synaptic vesicles were prepared from rat brain cortex by standards methods (Huttner et al., 1983). BON clones were cultured on $15 \mathrm{~cm}$ dishes. For each clone, cells from three confluent dishes $\left(\sim 10^{8}\right.$ cells $)$ were washed with PBS, scraped, and recovered in $10 \mathrm{ml}$ of chilled $0.32 \mathrm{M}$ sucrose and $4 \mathrm{~mm}$ HEPES-KOH, pH 7.4. Cells were homogenized using a Bioneb Cell Disruption System (Glas-Col Laboratory Products). A single cycle of nebulization under a $3.3 \mathrm{l} / \mathrm{min}$ stream of nitrogen at 2.5 bar was sufficient to break virtually all cells but maintained the latency of an intralysosomal marker ( $\beta$-glucuronidase, measured using 4-methylumbelliferyl- $\beta$-D-glucuronide as substrate) at $\sim 60 \%$. Nuclei and cell debris were pelleted at $10,000 \times g$ for $5 \mathrm{~min}$, and the resulting supernatant was centrifuged at $200,000 \times g$ for $20 \mathrm{~min}$. The resulting membrane pellet was resuspended in $400 \mu \mathrm{l}$ of ice-cold $0.32 \mathrm{M}$ sucrose, 4 $\mathrm{mm} \mathrm{KCl,} 4 \mathrm{~mm} \mathrm{MgSO}_{4}$, and $10 \mathrm{~mm} \mathrm{HEPES}-\mathrm{KOH}, \mathrm{pH} 7.4$ (final concentration, $10 \mathrm{mg} / \mathrm{ml}$ protein). $\left[{ }^{3} \mathrm{H}\right] \mathrm{L}$-glutamate and $\left[{ }^{3} \mathrm{H}\right] \mathrm{L}$-aspartate (both from Amersham Pharmacia Biotech, Uppsala, Sweden) uptake assays were performed as described previously (Bellocchio et al., 2000). Each uptake measurement was performed in triplicate and is expressed as mean \pm SEM. Experiments were independently repeated twice on two different BON-DNPI clones.

Immunoblotting. Anti-VGLUT1 antiserum was obtained by immunizing rabbits (Agro-Bio, Villeny, France) against the peptide CGLAPSYGATHSTVQPPR coupled via its cysteine residue to keyhole limpet hemocyanin (KLH). For the anti-DNPI serum, rabbits were immunized against the peptide HEDELDEETGDITQNYINY coupled at its $\mathrm{N}$ terminus to KLH. Western blots were performed with 5 or $10 \mu \mathrm{g}$ of protein per lane (see Figs. 2 and 3, respectively). Immunoreactivity was detected with anti-DNPI (1:1000), anti-VGLUT1 (1:3000), or antiVIAAT (1:5000) (Dumoulin et al., 1999) sera by chemiluminescence.

In situ hybridization. Regional in situ hybridization was performed with antisense $\left[{ }^{35} \mathrm{~S}\right] \mathrm{dATP}$-labeled oligonucleotides 5'-GCACTGGGCACAAGGGAAGACTTGCATCTT-3' and 5' -ACAGATTGCACTTGATGGGACTCTCACGGT-3' for VGLUT1 and DNPI, respectively, as described previously (Sagne et al., 2001). Cold in situ hybridization was performed with antisense riboprobes labeled with either fluorescein-UTP (for VGLUT1) or digoxigenin-UTP (for DNPI) (Ravassard et al., 1997). Colorimetric revelations were obtained with 5-bromo-4chloro-3-indolyl phosphate (Roche Products, Hertforshire, UK) and either nitroblue tetrazolium (Roche) or 2-[4-iodophenyl]-3-[4-nitrophenyl]5-phenyl-tetrazolium chloride (Roche) for the blue and red staining, respectively.

Immunoautoradiography. Immunoautoradiography was performed as described previously (Masson et al., 1999). In brief, sagittal or coronal rat brain sections were fixed with $4 \%$ paraformaldehyde and incubated with each antiserum (1:2000 dilution) and then with [ $\left.{ }^{125} \mathrm{I}\right] \mathrm{IgG}(0.25 \mu \mathrm{Ci} / \mathrm{ml}$; Amersham Pharmacia Biotech). Sections were apposed to x-ray films ( $\beta$ max; Amersham Pharmacia Biotech) for $5 \mathrm{~d}$.

Immunohistochemistry. Sprague Dawley rats were deeply anesthetized with sodium chloral hydrate and perfused transcardially with a mixture of $2 \%$ paraformaldehyde and $0.2 \%$ glutaraldehyde. Frontal sections of the brain were cut on a vibrating microtome at $70 \mu \mathrm{m}$, collected in PBS, cryoprotected, freeze-thawed, and stored in PBS until use.
VGLUT1 and DNPI were codetected according to an immunofluorescence method that permits the simultaneous detection of two antibodies raised in the same species (Shindler and Roth, 1996). VGLUT1 antiserum (1:2000) was detected with the tyramide system (TSA) (NEN, Boston, MA). Conventional fluorescently labeled secondary antibodies detected DNPI antiserum (1:3000). For the last step of the experiment, the sections were incubated in a mixture of streptavidin coupled to fluorescein (1:1000; Jackson ImmunoResearch, West Grove, PA) to complete the detection of VGLUT1 by the TSA method and goat anti-rabbit coupled to cyanine-3 (CY3) (1:200; Jackson ImmunoResearch) to detect DNPI. The sections were mounted in Vectashield (Vector Laboratories, Burlingame, CA) and observed using a confocal microscope (LSM 410; Zeiss, Oberkochen, Germany). The specificity of each labeling was proven by the absence of DNPI or VGLUT1 labeling when the primary or secondary antibody was omitted. An antisynaptophysin monoclonal serum (1:500; Chemicon, Temecula, CA) was used for immunofluorescence codetection of DNPI, VGLUT1, and synaptophysin, visualized with anti-rabbit IgG-CY3 or anti-mouse IgGFITC conjugates (Jackson ImmunoResearch).

VGLUT1 and DNPI were detected at the electron microscopic level using the preembedding immunogold method with silver intensification (Bernard et al., 1999). After treatment with 1\% osmium, dehydration, and embedding in resin, ultrathin sections were cut, stained with lead citrate, and examined using a Philips CM10 EM.

\section{RESULTS \\ DNPI is a glutamate transporter}

To investigate the molecular function of DNPI, the neuroendocrine cell line BON (Evers et al., 1991) was stably transfected with a DNPI expression plasmid. The ability of a membrane fraction enriched in DNPI (data not shown) to accumulate $\left[{ }^{3} \mathrm{H}\right] \mathrm{L}$ glutamate in the presence of ATP was examined. For comparison, membranes from BON clones expressing VGLUT1 or, as negative controls, VIAAT (McIntire et al., 1997; Sagne et al., 1997) or no cDNA, were analyzed in parallel experiments. Membranes from DNPI-expressing cells (Fig. 1 $A$ ) and VGLUT1expressing cells (data not shown) accumulated twice as much $\left[{ }^{3} \mathrm{H}\right]$ glutamate than negative controls. $\left[{ }^{3} \mathrm{H}\right]$ glutamate accumulation was linear over time for $3 \mathrm{~min}$ and reached a plateau level at $\sim 15$ min. As reported for VGLUT1 (Bellocchio et al., 2000; Takamori et al., 2000), the DNPI-dependent uptake of $\left[{ }^{3} \mathrm{H}\right]$ glutamate was inhibited by the $\mathrm{H}^{+}$ionophore carbonyl cyanide m-chlorophenylhydrazone (CCCP) $(50 \mu \mathrm{M})$ and by a saturating concentration $(5 \mu \mathrm{M})$ of Evans Blue (Fig. $1 B$ ), a competitive inhibitor of glutamate uptake into synaptic vesicles (Roseth et al., 1995). In contrast, the $\left[{ }^{3} \mathrm{H}\right]$ glutamate uptake activity endogenous to BON cells was insensitive to Evans Blue and only slightly inhibited by CCCP.

To characterize further the bioenergetics of this DNPImediated glutamate transport, several inhibitors were tested. For clarity, results are shown only for the CCCP-sensitive (DNPImediated) component of uptake (Fig. $1 C, D$ ). Bafilomycin A1, a selective inhibitor of the $\mathrm{H}^{+}$-translocating ATPase from acidic organelles, strongly inhibited $\left[{ }^{3} \mathrm{H}\right]$ glutamate uptake by DNPI, meaning that this uptake is driven by a transmembrane $\mathrm{H}^{+}$ electrochemical gradient $\left(\Delta \mu \mathrm{H}^{+}\right)$. Nigericin, an ionophore that disrupts transmembrane $\mathrm{pH}$ gradients by exchanging $\mathrm{H}^{+}$for $\mathrm{K}^{+}$, did not significantly inhibit the uptake $(89.1 \pm 9.6 \%$ of control; mean \pm SEM; three independent experiments on two BON clones), suggesting that the translocation of glutamate anions by DNPI is mainly driven by the electrical component of $\Delta \mu \mathrm{H}^{+}$. Indeed, a further addition of the $\mathrm{K}^{+}$ionophore valinomycin, to dissipate the remaining electrical gradient, inhibited $\left[{ }^{3} \mathrm{H}\right]$ glutamate uptake as efficiently as CCCP. Identical effects were observed for the VGLUT1-mediated glutamate uptake (Fig. 1C), as 

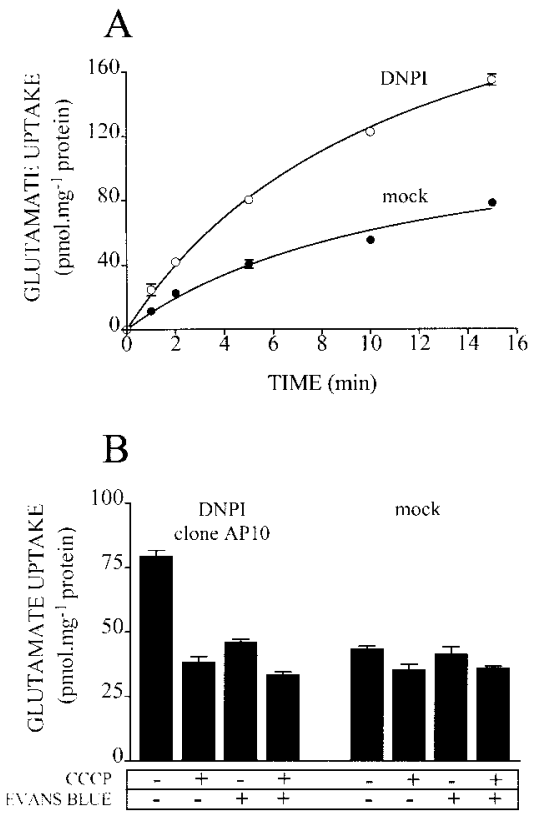

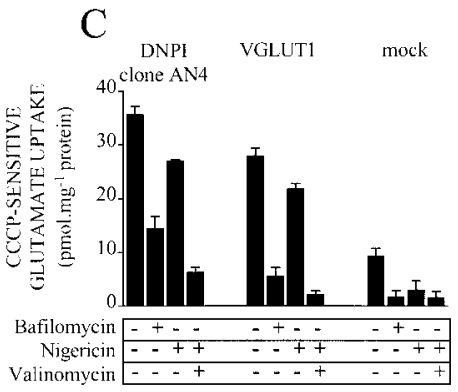

$\mathrm{D}$

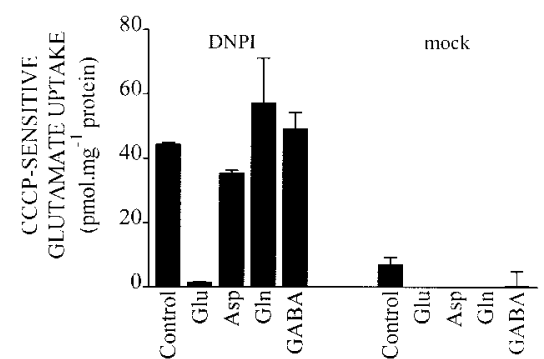

$\mathrm{E}$
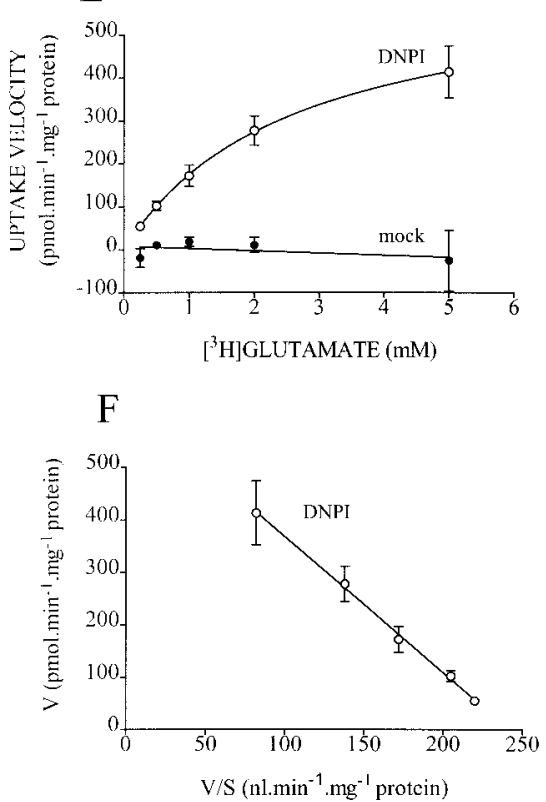

Figure 1. Functional expression of DNPI. Membranes from BON cells expressing DNPI or VGLUT1 were incubated at $30^{\circ} \mathrm{C}$ for 5 min $(B-D)$ with $44 \mu \mathrm{M}\left[{ }^{3} \mathrm{H}\right] \mathrm{L}$-glutamate $(A-D)$ and the following additives, as stated: $50 \mu \mathrm{M}$ CCCP, $5 \mu \mathrm{M}$ Evans Blue, $5 \mu \mathrm{M}$ nigericin, $20 \mu \mathrm{M}$ valinomycin, $10 \mathrm{~mm}$ L-glutamate $(G l u), 10 \mathrm{~mm}$ L-aspartate $(A s p), 10 \mathrm{~mm}$ L-glutamine $(G l n)$, or $10 \mathrm{~mm}$ GABA. Accumulated $\left[{ }^{3} \mathrm{H}\right] \mathrm{L}$-glutamate was determined in triplicate by filtration. Background uptake levels were determined with BON cells expressing no cDNA or the unrelated VIAAT cDNA [mock transfections (mock)]. In $A$, membranes were incubated with $44 \mu \mathrm{M}\left[{ }^{3} \mathrm{H}\right] \mathrm{L}$-glutamate for increasing periods of time. In $E$, membranes were incubated for 3 min with increasing concentrations of $\left[{ }^{3} \mathrm{H}\right] \mathrm{L}$-glutamate. $F$ is an Eadie-Hofstee representation of the DNPI curve shown in $E$.

reported previously (Bellocchio et al., 2000; Takamori et al., 2000).

The substrate selectivity of DNPI was assessed by adding 10 mM unlabeled amino acids simultaneously with $\left[{ }^{3} \mathrm{H}\right]$ glutamate in both the absence and presence of CCCP. Although L-glutamate strongly inhibited DNPI-mediated uptake (11.9 $\pm 4.5 \%$ of control residual activity), activities corresponding to $82 \pm 4,122 \pm 4$, and $111 \pm 0.8 \%$ of control were observed in the presence of L-aspartate, L-glutamine, and GABA, respectively $(n=3)$. Similarly, aspartate, glutamine, and GABA did not significantly inhibit VGLUT1 (data not shown), as reported previously (Bellocchio et al., 2000; Takamori et al., 2000). The selectivity for glutamate over aspartate was confirmed by the absence of significant $\left[{ }^{3} \mathrm{H}\right] \mathrm{L}$-aspartate accumulation into DNPI- or VGLUT1containing membranes (data not shown). Incubation of DNPIcontaining membranes with increasing concentrations of $\left[{ }^{3} \mathrm{H}\right]$ glutamate for $3 \mathrm{~min}$ showed that the transport velocity saturates (Fig. $1 E, F$ ), with $K_{\mathrm{M}}$ and $V_{\max }$ values of $1.9 \pm 0.4 \mathrm{~mm}$ and $470 \pm 110 \mathrm{pmol} \cdot \mathrm{min}^{-1} \cdot \mathrm{mg}^{-1}$ protein, respectively $(n=3)$. Similarly, values of $3.4 \pm 0.9 \mathrm{~mm}$ and $500 \pm 5 \mathrm{pmol} / \mathrm{min}$ protein were obtained for VGLUT1 $(n=2)$, in agreement with previous observations (Bellocchio et al., 2000). Evans Blue inhibited VGLUT1- and DNPI-mediated uptakes with similar $\mathrm{IC}_{50}$ values of $\sim 300 \mathrm{~nm}$ (data not shown).

Together, these data demonstrate that DNPI is a glutamate transporter that displays functional characteristics very similar, if not identical, to those of VGLUT1. Because DNPI also localizes to synaptic vesicles in brain (see below), the protein is renamed VGLUT2 hereafter.

\section{Regional distribution of VGLUTs}

The regional distributions of VGLUT1 and VGLUT2 transcripts were assessed by in situ hybridization. As shown in Figure $2 A$,

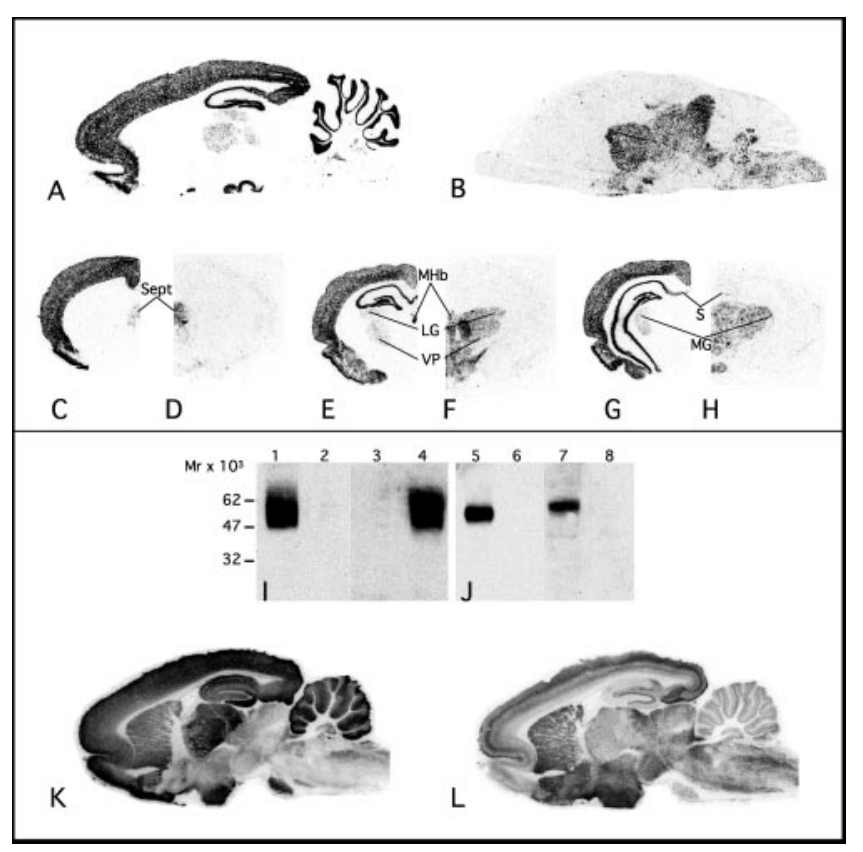

Figure 2. Regional distribution of VGLUT1 and VGLUT2. $A-H, \mathrm{Hy}-$ bridization of sagittal $(A, B)$ or coronal $(C-H)$ rat brain sections with antisense $\left[{ }^{35}\right.$ S]oligonucleotides deduced from VGLUT1 $(A, C, E, G)$ or $\operatorname{VGLUT2}(B, D, F, H)$ sequences. $I, J$, Immunoblot analysis of BON cells stably expressing VGLUT1 (lanes 1,3) or VGLUT2 (lanes 2, 4) and of total brain extracts $(J)$. Anti-VGLUT1 (lanes 1, 2, 5), anti-VGLUT2 (lanes 3, 4, 7), or preimmune (lanes 6, 8) sera were used. $K, L$, Immunoautoradiograms of sagittal sections performed with anti-VGLUT1 $(K)$ and anti-VGLUT2 $(L)$ sera. $L G$, Lateral geniculate thalamus nucleus; $M G$, medial geniculate thalamus nucleus; $M H b$, medial habenula; $S$, subiculum; Sept, septum; VP, ventroposterior thalamic nucleus. 
Figure 3. VGLUT2 localizes to synaptic vesicles. $A$, Immunoblot analysis of rat brain subcellular fractions. Abbreviations are from Huttner et al. (1983). VGLUT2 is enriched in synaptosomes $(P 2)$ and synaptic vesicles $(L P 2) . B$, Ultrastructural localization of VGLUT2 and VGLUT1. Immunoparticles are detected over synaptic vesicle clusters in axonal terminals $(T)$ making asymmetrical synaptic contacts (arrows) with dendritic shafts $(d)$ or spines $(s)$. Scale bars: $B$, Cerebellum, $500 \mathrm{~nm} ; B$, Cortex, $250 \mathrm{~nm}$. $C$, Immunofluorescent detection of VGLUT1 or VGLUT2 (both in red) and synaptophysin (in green) are shown in the striatum. VGLUTs puncta colocalizes with synaptophysin (yellow). Note the low frequency of red puncta. $c c$, Corpus callosum. Scale bar, $25 \mu \mathrm{m}$.
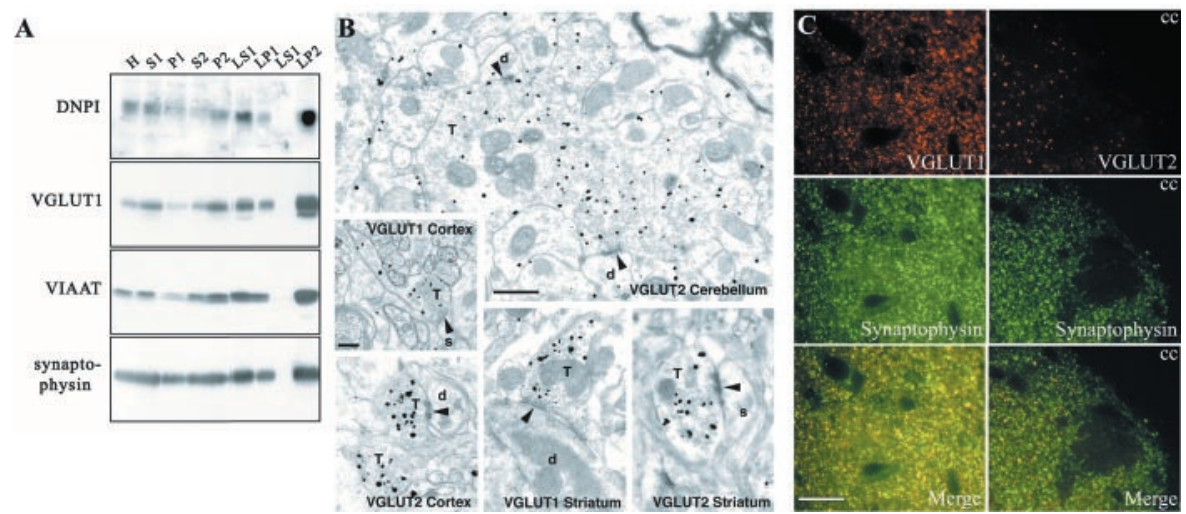

VGLUT1 mRNA is highly enriched in olfactory bulb, cerebral and cerebellar cortices, medial habenula, pontine nucleus (data not shown), and hippocampus (including the subiculum). A lower level of labeling was detected in lateral septum and thalamic nuclei. On the other hand, VGLUT2 transcript is strongly expressed in all thalamus nuclei (except the reticular nucleus), in hypothalamus, inferior and superior colliculi, deep cerebellar nuclei, and many neurons from the brainstem (Fig. 2B). Moderate to low levels of VGLUT2 mRNA were observed in hippocampus, some cortical areas, and amygdala (data not shown). These distributions, which are consistent with previous reports (Ni et al., 1995; Hisano et al., 2000), thus appear mostly complementary. However, a more careful examination of coronal brain sections revealed a regional colocalization in some thalamic nuclei (for example, lateral and medial geniculate, ventral posterior), lateral habenula, septum, and subiculum (Fig. $2 \mathrm{C}-\mathrm{H}$ ).

The distribution of VGLUT proteins was examined using polyclonal antisera. Antiserum specificity was first assessed by immunoblotting (Fig. 2I,J). Each antiserum specifically recognized a single diffuse $\sim 60 \mathrm{kDa}$ protein in extracts from BON cells expressing VGLUT1 (lane 1) or VGLUT2 (lane 4). No crossreactivity was observed (lanes 2, 3). A single band was also detected by each antiserum in brain extracts (lanes 5, 7). These bands disappeared when preimmune serum was used (lane 6,8). These results show that our VGLUT1 and VGLUT2 antisera are highly specific, a conclusion further supported by immunofluorescence analysis of transfected BON and COS cells (data not shown).

Immunoautoradiographic analysis using these antisera revealed that most brain areas coexpress both transporters, with a somewhat more laminar pattern for VGLUT2 than for VGLUT1 (Fig. $2 K, L)$. This is in complete contrast to the distribution of their cognate transcripts. For example, although no VGLUT2 mRNA was detected in cerebral cortex (Fig. 2B), VGLUT2 immunoreactivity was strong in cortical layers IV and VI. These data suggested that the VGLUT2 protein is targeted to nerve terminals of projection neurons, as reported for VGLUT1 (Bellocchio et al., 1998).

\section{VGLUT2 localizes to synaptic vesicles at excitatory synapses}

The intracellular distribution of VGLUT2 was first examined by subcellular fractionation. As shown in Figure $3 A$, VGLUT2 copurifies with several established markers of synaptic vesicles, such as VGLUT1, VIAAT, and synaptophysin. The association to synaptic vesicles was confirmed by the accumulation of VGLUT2 at nerve terminals. As demonstrated by immunofluorescence microscopy, performed in the striatum, VGLUT2 punctuate labeling colocalizes with synaptophysin (Fig. 3C). Similar results were observed in all areas inspected (data not shown). The distribution of VGLUT2 was also examined at the ultrastructural level using a preembedding immunogold method. In cortex, cerebellum, and striatum, VGLUT2 immunoparticles accumulated over synaptic vesicle clusters at terminal boutons forming asymmetrical contacts with dendritic shafts or spines (Fig. 3B). These morphological features are classically displayed by excitatory synapses. No immunoparticles could be detected on symmetric putative GABAergic synapse (data not shown).

Combined biochemical, anatomical, and ultrastructural results strongly suggest that VGLUT2 localizes to synaptic vesicles at excitatory synapses, as does VGLUT1 (Fig. 3) (Bellocchio et al., 1998). These results are consistent with the conclusions of two recent studies (Fujiyama et al., 2001; Sakata-Haga et al., 2001). The expression of VGLUT2 at mossy fiber terminals in the cerebellum, which are established as glutamatergic, further confirmed this conclusion (Fig. 3B, top panel).

\section{VGLUT1 and VGLUT2 define distinct subsets of glutamatergic neurons}

We then examined whether VGLUT1 and VGLUT2 are coexpressed in glutamatergic neurons and/or nerve endings. This issue was first investigated by in situ hybridization, using colorimetric detection of VGLUT1 and VGLUT2 mRNA probes at the cellular level. Three classes of glutamatergic neuronal cell bodies could be observed, as illustrated for adjacent nuclei in a single section (Fig. 4A). VGLUT1-positive neurons (red) were observed in CA3 and in the amygdalo-hippocampal complex. Next, the medial amygdaloid nucleus only expressed the VGLUT2 mRNA (blue). Finally, both messengers were expressed in individual neurons of the lateral olfactory tract (red and blue cells). Additional cases of VGLUT1 and VGLUT2 coexpression were found. For instance, in the thalamus, two populations were present: VGLUT2-positive cells (data not shown) and VGLUT1VGLUT2-expressing cells (Fig. 4B,C).

The possible coexpression of both proteins at individual nerve terminals was also examined by double-immunofluorescence microscopy. However, we were not able to detect "mixed" VGLUT1-VGLUT2-positive terminals in projection areas from these neurons. As illustrated in hippocampus (Fig. 4D) and cerebral cortex (Fig. 4E), VGLUT1 immunoreactivity (green) never merged with that of VGLUT2 (red).

Together, these data show that the VGLUTs define three subsets of glutamatergic neurons and at least two subsets of glutamatergic terminals. 


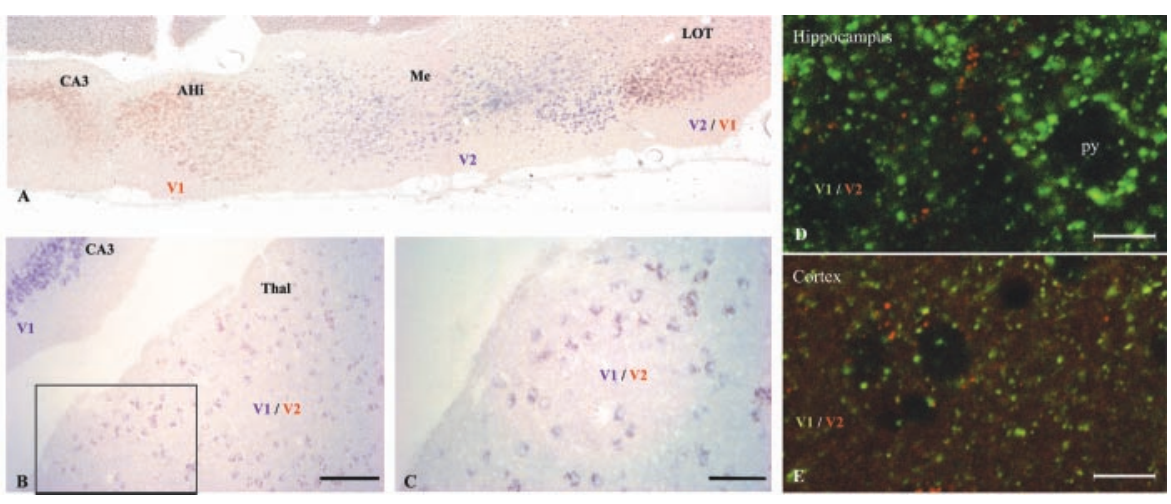

Figure 4. VGLUT1 and VGLUT2 define distinct subsets of glutamatergic neurons. $A$, Doublelabeling in situ hybridization allows the detection of three groups of neurons: VGLUT1-expressing (V1; red), VGLUT2-expressing (V2; blue), and VGLUT1-VGLUT2-expressing (V2/V1; blue and red staining) cells. Parasagittal section at lateral coordinate $\sim 2.4 \mathrm{~mm}$. $B, C$, VGLUT1-VGLUT2positive neurons are shown in the thalamus. Scale bars: $B, 200 \mu \mathrm{m} ; C, 100 \mu \mathrm{m}$. $D, E$, Double immunofluorescence with VGLUT1 (V1; green) and VGLUT2 (V2; red) antibodies detected by laser confocal microscopy. No colabeling was detected. Ahi, Amygdalohippocampal area; $C A 3$, field CA3 of hippocampus; $L O T$, lateral olfactory tract nucleus; $M e$, medial amygdaloid nucleus; $p y$, pyramidal cell. Scale bars, $10 \mu \mathrm{m}$.

\section{DISCUSSION}

In this study, we show that a novel member of the type $\mathrm{I} \mathrm{Na}^{+} / \mathrm{Pi}$ cotransporter family, DNPI, is indeed a new vesicular glutamate transporter (VGLUT2). First, the protein biochemically and anatomically localizes to synaptic vesicles (Fig. 3). Second, VGLUT2 mediates a glutamate uptake activity that is very similar, if not identical, to the activity of native synaptic vesicles or recombinant VGLUT1 (Bellocchio et al., 2000; Takamori et al., 2000). Finally, VGLUT2 is expressed in a subset of presynaptic excitatory terminals (Fig. $3 B$ ).

Although the two transporters exhibited similar activities, VGLUT1- and VGLUT2-positive neurons displayed strongly different distributions (Fig. 2), implying that the expression of these two genes is regulated by distinct mechanisms. This was particularly clear when the regional distribution of VGLUT1- and VGLUT2 mRNA was examined. A high complementarity was observed, with only a partial overlap (Fig. $2 A-H$ ). Investigation at the cellular level confirmed the existence of neurons expressing either VGLUT1 or VGLUT2. However, these experiments also detected an additional subset of neurons that expresses both transcripts (Fig. 4A-C). Therefore, the VGLUTs define at least three subsets of glutamatergic neurons, expressing the type 1 , the type 2, or both transcripts.

The same analysis was also performed at the level of glutamatergic terminals, by comparing the microscopic distributions of VGLUT1 and VGLUT2 immunoreactivities (Fig. 4D,E). However, in this case, only two classes of glutamatergic terminals were found, expressing either the type 1 or the type 2 protein, even in projection areas of regions containing mixed VGLUT1-VGLUT2 mRNA-expressing neurons. Three hypotheses can be proposed at this stage. The most plausible explanation is that we failed to detect colabeled terminals because of their rare occurrence compared with isoform-specific terminals. A second possibility would be that mixed glutamatergic neurons express either the VGLUT1 or the VGLUT2 protein, although both transcripts are present in the soma. Finally, a third provocative alternative would be that both proteins are synthesized in VGLUT1-VGLUT2-positive cells, but an unknown mechanism ensures that they are targeted to distinct terminals within the same neuron.

It must be noted that, although VGLUT1 and VGLUT2 are highly homologous, their amino acid sequences strongly differ at their C termini. In particular, VGLUT1 exhibit proline-rich motifs that are lacking in VGLUT2. Therefore, VGLUT1 and VGLUT2 may interact with distinct sets of associated proteins. This could have important functional consequences at several levels, including protein targeting. The existence of two VGLUTs should thus provide important tools for the anatomical and functional characterization of glutamatergic pathways.

\section{REFERENCES}

Aihara Y, Mashima H, Onda H, Hisano S, Kasuya H, Hori T, Yamada S, Tomura H, Yamada Y, Inoue I, Kojima I, Takeda J (2000) Molecular cloning of a novel brain-type $\mathrm{Na}(+)$-dependent inorganic phosphate cotransporter. J Neurochem 74:2622-2625.

Bellocchio EE, Hu H, Pohorille A, Chan J, Pickel VM, Edwards RH (1998) The localization of the brain-specific inorganic phosphate transporter suggests a specific presynaptic role in glutamatergic transmission. J Neurosci 18:8648-8659.

Bellocchio EE, Reimer RJ, Fremeau Jr RT, Edwards RH (2000) Uptake of glutamate into synaptic vesicles by an inorganic phosphate transporter. Science 289:957-960.

Bernard V, Levey AI, Bloch B (1999) Regulation of the subcellular distribution of $\mathrm{m} 4$ muscarinic acetylcholine receptors in striatal neurons in vivo by the cholinergic environment: evidence for regulation of cell surface receptors by endogenous and exogenous stimulation. J Neurosci 19:10237-10249.

Dent JA, Davis MW, Avery L (1997) avr-15 encodes a chloride channel subunit that mediates inhibitory glutamatergic neurotransmission and ivermectin sensitivity in Caenorhabditis elegans. EMBO J 16:5867-5879.

Dumoulin A, Rostaing P, Bedet C, Levi S, Isambert MF, Henry JP, Triller A, Gasnier B (1999) Presence of the vesicular inhibitory amino acid transporter in GABAergic and glycinergic synaptic terminal boutons. J Cell Sci 112:811-823.

Evers BM, Townsend Jr CM, Upp JR, Allen E, Hurlbut SC, Kim SW, Rajaraman S, Singh P, Reubi JC, Thompson JC (1991) Establishment and characterization of a human carcinoid in nude mice and effect of various agents on tumor growth. Gastroenterology 101:303-311.

Fujiyama F, Furuta T, Kaneko T (2001) Immunocytochemical localization of candidates for vesicular glutamate transporters in the rat cerebral cortex. J Comp Neurol 435:379-387.

Gasnier B (2000) The loading of neurotransmitters into synaptic vesicles. Biochimie 82:327-337.

Hisano S, Hoshi K, Ikeda Y, Maruyama D, Kanemoto M, Ichijo H, Kojima I, Takeda J, Nogami H (2000) Regional expression of a gene encoding a neuron-specific $\mathrm{Na}(+)$-dependent inorganic phosphate cotransporter (DNPI) in the rat forebrain. Brain Res Mol Brain Res $83: 34-43$.

Huttner WB, Schiebler W, Greengard P, De Camilli P (1983) Synapsin I (protein I), a nerve terminal-specific phosphoprotein. III. Its association with synaptic vesicles studied in a highly purified synaptic vesicle preparation. J Cell Biol 96:1374-1388.

Lee RY, Sawin ER, Chalfie M, Horvitz HR, Avery L (1999) EAT-4, a homolog of a mammalian sodium-dependent inorganic phosphate cotransporter, is necessary for glutamatergic neurotransmission in $\mathrm{Cae}$ norhabditis elegans. J Neurosci 19:159-167.

Masson J, Cervera P, Cote S, Morisette J, Aidouni Z, Giros B, Hamon M, Falardeau P, Mestikawy SE (1999) Characterization and distribution of $\mathrm{Hxt1}$, a $\mathrm{Na}(+) / \mathrm{Cl}(-)$-dependent orphan transporter, in the human brain. J Neurosci Res 56:146-159.

McIntire SL, Reimer RJ, Schuske K, Edwards RH, Jorgensen EM (1997) Identification and characterization of the vesicular GABA transporter. Nature 389:870-876.

Ni B, Rosteck Jr PR, Nadi NS, Paul SM (1994) Cloning and expression of a cDNA encoding a brain-specific $\mathrm{Na}(+)$-dependent inorganic phosphate cotransporter. Proc Natl Acad Sci USA 91:5607-5611.

Ni B, Wu X, Yan GM, Wang J, Paul SM (1995) Regional expression and cellular localization of the $\mathrm{Na}^{+}$-dependent inorganic phosphate cotransporter of rat brain. J Neurosci 15:5789-5799. 
Ravassard P, Chatail F, Mallet J, Icard-Liepkalns C (1997) Relax, a novel rat bHLH transcriptional regulator transiently expressed in the ventricular proliferating zone of the developing central nervous system. J Neurosci Res 48:146-158.

Roseth S, Fykse EM, Fonnum F (1995) Uptake of L-glutamate into rat brain synaptic vesicles: effect of inhibitors that bind specifically to the glutamate transporter. J Neurochem 65:96-103.

Sagne C, El Mestikawy S, Isambert MF, Hamon M, Henry JP, Giros B, Gasnier B (1997) Cloning of a functional vesicular GABA and glycine transporter by screening of genome databases. FEBS Lett 417:177-183.

Sagne C, Agulhon C, Ravassard P, Darmon M, Hamon M, El Mestikawy S, Gasnier B, Giros B (2001) Identification and characterization of a lysosomal transporter for small neutral amino acids. Proc Natl Acad Sci USA 98:7206-7211.
Sakata-Haga H, Kanemoto M, Maruyama D, Hoshi K, Mogi K, Narita M, Okado N, Ikeda Y, Nogami H, Fukui Y, Kojima I, Takeda J, Hisano S (2001) Differential localization and colocalization of two neuron-types of sodium-dependent inorganic phosphate cotransporters in rat forebrain. Brain Res 902:143-155.

Shindler KS, Roth KA (1996) Double immunofluorescent staining using two unconjugated primary antisera raised in the same species. J Histochem Cytochem 44:1331-1335.

Takamori S, Rhee JS, Rosenmund C, Jahn R (2000) Identification of a vesicular glutamate transporter that defines a glutamatergic phenotype in neurons. Nature 407:189-194.

Werner A, Dehmelt L, Nalbant P (1998) Na+-dependent phosphate cotransporters: the NaPi protein families. J Exp Biol 201:3135-3142. 